\title{
YJIT: A Basic Block Versioning JIT Compiler for CRuby
}

\author{
Maxime Chevalier-Boisvert \\ Shopify \\ Canada \\ maxime.chevalierboisvert@shopify.com \\ Si Xing (Alan) Wu
Shopify
Canada
paper@alanwu.email
}

\author{
Noah Gibbs \\ Shopify \\ United Kingdom \\ noah.gibbs@shopify.com \\ Aaron Patterson \\ Shopify \\ United States \\ aaron.patterson@shopify.com
}

\author{
Jean Boussier \\ Shopify \\ France \\ jean.boussier@shopify.com \\ Kevin Newton \\ Shopify \\ United States \\ kevin.newton@shopify.com
}

\begin{abstract}
Ruby is a dynamically typed programming language with a large breadth of features which has grown in popularity with the rise of the modern web, and remains at the core of the implementation of many widely-used websites.

CRuby, the default implementation of the language, features a JIT compiler known as MJIT, but developers often do not enable it in production environments, because it does not always yield performance improvements on real-world software. Attempts to independently reimplement the Ruby language, such as JRuby and TruffleRuby have shown impressive performance results on benchmarks, but often lag behind CRuby when it comes to supporting new additions to the language, which limits their adoption.

We introduce YJIT, a new JIT compiler built inside CRuby based on a Lazy Basic Block Versioning (LBBV) architecture. We show that while our compiler does not match the peak performance of TruffleRuby, it offers near-100\% compatibility with existing Ruby code, impressively fast warmup, and speedups from $15 \%$ to $19 \%$ on sizeable benchmarks based on real-world software.
\end{abstract}

CCS Concepts: • Software and its engineering $\rightarrow$ Justin-time compilers.

This work is licensed under a Creative Commons Attribution 4.0 International License.

VMIL '21, October 19, 2021, Chicago, IL, USA

(C) 2021 Copyright held by the owner/author(s).

ACM ISBN 978-1-4503-9109-2/21/10.

https://doi.org/10.1145/3486606.3486781
Keywords: just-in-time, compiler, dynamically typed, optimization, bytecode, ruby

ACM Reference Format:

Maxime Chevalier-Boisvert, Noah Gibbs, Jean Boussier, Si Xing (Alan) Wu, Aaron Patterson, Kevin Newton, and John Hawthorn. 2021. YJIT: A Basic Block Versioning JIT Compiler for CRuby. In Proceedings of the 13th ACM SIGPLAN International Workshop on Virtual Machines and Intermediate Languages (VMIL '21), October 19, 2021, Chicago, IL, USA. ACM, New York, NY, USA, 8 pages. https://doi.org/10.1145/3486606.3486781

\section{Introduction}

Ruby is a programming language featuring dynamic typing and garbage collection, as well as both object-oriented and functional programming constructs. The language, which takes inspiration from Perl and Smalltalk, has grown in popularity with the rise of the modern web, and remains at the core of the implementation of many widely-used websites.

The feature-rich and highly dynamic nature of Ruby make it challenging to optimize. CRuby, the official Ruby implementation, includes a JIT compiler known as MJIT, but despite impressive numbers on synthetic benchmarks, MJIT has not, thus far, yielded speedups in production workloads. Large-scale enterprise users such as Shopify and GitHub typically leave it disabled, relying on the CRuby interpreter only.

There are ongoing efforts, such as JRuby and TruffleRuby, to produce alternative Ruby implementations. These have shown great peak performance numbers, but for a variety of reasons, both practical and political, real-world deployments are still very limited. The current situation mirrors that of alternative reimplementations of other existing languages. TruffleRuby, JRuby, PyPy and LuaJIT are all impressive implementation efforts with attractive performance numbers, but there is often a gap in terms of compatibility with and 
support for all the features of the latest reference implementation. This gap makes it challenging for users to switch.

YJIT is an open source ${ }^{1}$ development effort being led at Shopify, with active participation from developers at GitHub, to build a new JIT compiler for Ruby inside the reference CRuby implementation. Retrofitting a new JIT compiler into an older codebase which was not originally designed with JIT compilation in mind presents its own set of challenges, however, it also comes with some important benefits. Namely, YJIT is nearly $100 \%$ compatible with the latest CRuby as well as all existing Ruby packages, and it is practically a drop-in replacement for existing CRuby binaries, making deployment very straightforward. As of today, YJIT has been able to pass all of the nearly 30,000 CRuby unit tests, and to match the reference CRuby implementation in all of the tests in the Shopify and GitHub continuous integration (CI) test suites.

The architecture of YJIT is based on a Lazy Basic Block Versioning (LBBV) JIT compiler architecture [6, 7]. This workin-progress paper provides clear evidence that such an architecture can realistically scale to large, real-world software, providing both fast warmup times and significant speedups.

Our contributions are:

- An open source implementation of YJIT, a JIT compiler based on LBBV for the Ruby programming language which is highly compatible with existing Ruby code.

- Empirical evidence that a JIT compiler based on an LBBV architecture can deliver fast warmup times on benchmarks based on real-world software.

- Empirical evidence that YJIT produces speedups relative to the current CRuby interpreter and MJIT on benchmarks based on real-world software.

Section 2 of this paper delves into some background about Ruby and its reference implementation. Section 3 surveys key details of the architecture and implementation of YJIT. Section 4 looks at performance results and warmup time on benchmarks. Finally, section 5 surveys related work and section 6 outlines directions for future work.

\section{Background}

\subsection{The Ruby Programming Language}

Ruby has a very broad set of features and makes JavaScript look minimalistic by comparison. For instance, there are four different types of executable objects in Ruby: methods, blocks, procs, and lambdas. This broad set of features, in and of itself, makes implementing the language more challenging. There is more implementation work, and there are more potential edge cases to consider.

Ruby method calls perform dynamic dispatch. Methods can be dynamically redefined and the method lookup order can also be dynamically changed. Common operations

\footnotetext{
$\overline{{ }^{1} \text { https://github.com/Shopify/yjit }}$
}

such as integer arithmetic are not primitive operations in the language and are instead calls to methods which can be redefined at run-time. Ruby has support for first-class environments which allow local variable access from outside the local scope. That is, callees can redefine local variables in their callers. This can make a number of compiler optimizations more challenging to implement correctly.

\subsection{The CRuby Virtual Machine}

The core of CRuby is a stack-based interpreter, which executes YARV bytecode instructions [17] one at a time, with each instruction able to push and pop values off of the temporary stack. A second separate stack is used to keep track of control frame objects corresponding to activation records for method calls. We will mention specific aspects that are relevant to this work, but the design of the CRuby VM is explained in great detail in the book Ruby Under a Microscope [19].

CRuby uses a system of tag bits to differentiate small immediate values such as fixnums (63-bit integers), as well as the constants true, false, and nil from heap objects, and a generational mark and sweep garbage collector which has the ability to move objects in memory.

Ruby, taking inspiration from Smalltalk, makes heavy use of method calls. Internally, there are multiple different types of method calls, including calls to Ruby methods, calls to blocks (a special kind of closure), and calls to methods that are implemented in C. CRuby internally categorizes methods into 11 different types, each with a slightly different implementation of the send instruction [19]. This makes optimization work difficult because the send operation cannot easily be reduced to one single atomic primitive.

\section{$2.3 \quad$ MJIT}

MJIT compiles Ruby source by generating $\mathrm{C}$ source code. It calls an external $\mathrm{C}$ compiler, then loads the output via dynamic linking. Outputting $\mathrm{C}$ source code allows MJIT to reuse parts of the interpreter's source in its output where appropriate, allowing for ease of development. It uses a background thread to generate $\mathrm{C}$ code and waits for the external C compiler to produce a shared library.

MJIT translates Ruby methods to $\mathrm{C}$ functions largely by concatenating the interpreter's implementation of bytecode instructions. Branch instructions are mapped to $\mathrm{C}$ control flow constructs and method call instructions have special handling. MJIT uses the same monomorphic inline cache structure as the interpreter to specialize call sites. For select callees that are leaves (do not make further calls), MJIT is able to avoid creating a stack frame. Calls to other compiled methods are done through a function pointer.

MJIT benefits from optimizations implemented in the $\mathrm{C}$ compiler. Additionally, using $\mathrm{C}$ means MJIT can target different machine architectures relatively easily. However, using 
an external compiler means MJIT does not have full control over compilation time. MJIT currently uses the same $\mathrm{C}$ compiler used to build the interpreter, which might have unsatisfactory performance in the context of JIT compilation. Lack of control over code generation details also makes implementing techniques such as code patching difficult, limiting optimization opportunities.

\subsection{Lazy Basic Block Versioning}

YJIT is built on an architecture based on LBBV, as described in previous works $[6,7]$. This architecture aims to provide fast compilation times while generating relatively efficient machine code when compared to a traditional baseline JIT compiler, without relying on expensive fixed-point program analyses and transformations.

Code for methods is compiled incrementally, one basic block at a time, as opposed to method-based compilers which treat whole methods as compilation units. LBBV can be seen as a form of lazy evaluation applied to code generation. This is achieved by using stubs and code patching. Code generation proceeds for as long as the compiler can determine the direction of a branch. When a branch whose direction cannot be determined is encountered, branch stubs are installed, and execution is resumed. Hitting a stub in turn interrupts execution to resume code generation.

LBBV interleaves code generation and execution, which opens up opportunities to specialize code based on the types, values, and execution paths that are encountered at run-time. Code that is not executed is never compiled. Machine code is appended to an array, and stubs are generated in a separate out-of-line (not inline) memory region so that they do not impact the instruction cache when they are not executed. The resulting generated code tends to be fairly linear and follows the natural flow of execution seen at run-time, helping reduce the occurrence of branch mispredictions.

To prevent a code size explosion due to the generation of multiple versions of basic blocks, YJIT relies on the same simple scheme described in [6], that is, we allow a fixed, maximum number of versions to be generated for each block (currently set to 4 by default). The last version generated must always be generic, in that it can accept any incoming value types.

\section{Architecture of YJIT}

Currently, YJIT only targets Linux and macOS platforms and x86-64 CPUs. This is in part motivated by the desire to prototype quickly, but also because the servers that run Shopify and GitHub's software are currently x86-64 machines running Linux. Future versions may add ARM64 support. YJIT supports all Ruby 3 features.

\subsection{Integration with CRuby}

YJIT uses CRuby's direct-threaded interpreter as a baseline. A counter monitors how many times a given method is called, and when a threshold is hit (currently 10 calls by default), YJIT begins incrementally generating code for said method. A JIT entry point is installed, which all future calls to the method will dispatch to. Generated code for methods with branches may include stubs that are only executed when a given target of a branch is hit. These stubs call back into YJIT (see Figure 1), which lazily generates more code on demand, patches the stub, and returns control to the newly generated code.

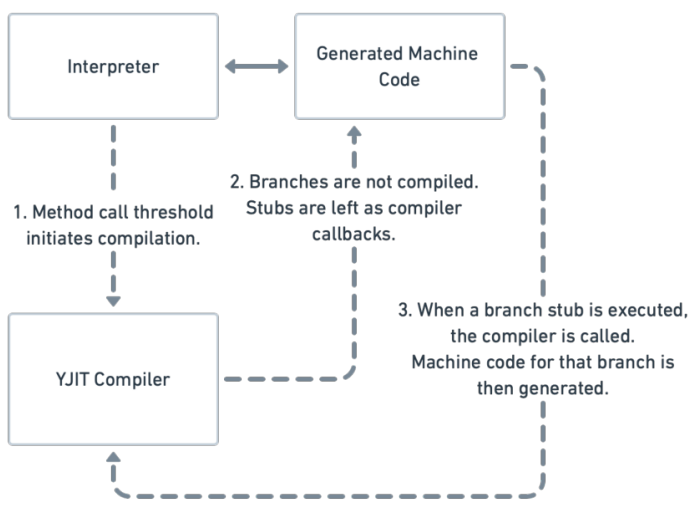

Figure 1. YJIT Compilation Pipeline.

The CRuby bytecode is rich and complex, and YJIT does not implement every operation on every type. Guards are used to specialize the fast path for various operations. When guards fail, YJIT can exit back to the interpreter, even in the middle of a method. This is done by restoring the interpreter's stack pointer and program counter, and then returning control to the interpreter. During development, statistics are routinely gathered to establish which operations cause us to exit to the interpreter most frequently, which guides our development, with the goal of maximizing time spent in code we generate.

For the sake of simplicity, YJIT uses the same calling convention and stack frame layout as CRuby, which makes entering and leaving generated code relatively simple. At this stage, YJIT also uses the CRuby stack to store temporary values, and has no register allocator. This is far from optimal, but it simplifies interfacing with the CRuby GC, and we believe that the main source of overhead, at present, is the inefficiency of the CRuby calling convention (see Section 6).

\subsection{Deoptimization}

The architecture of YJIT allows fine-grained code invalidation at the level of individual basic blocks versions. That is, individual versions of a basic block can be invalidated if specific assumptions made at code generation time are found to 
be violated at run-time. We make use of this to speculate that operations such as integer addition and subtraction will not be redefined at run-time, that constants will not be redefined, and that methods we are calling will not be redefined.

\subsection{Run-Time Value Promotion}

We implemented a technique originally described by Armin Rigo and used in the Psyco VM for Python [13], then subsequently implemented in PyPy [2]. That is, YJIT can promote run-time values to compile-time constants, implementing the equivalent of Psyco's unlift operator. This is done by interrupting compilation and inserting a new stub which will then peek at the run-time value, and resume compilation once the value is known. The use of LBBV makes this relatively simple because our architecture naturally allows incrementally compiling subsets of a method.

YJIT relies on the promotion of run-time values to implement a mechanism that is functionally similar to polymorphic inline caches. When generating code for an instance variable read instruction, we peek at the run-time value and determine its class, so that we can generate specialized machine code for reading the requested property on the class that was encountered. If at run-time, an object of a new class is encountered, this causes a jump to a stub, which will call back in to the JIT to generate a specialized instance variable read for the new class, again using the same run-time value promotion mechanism. The same technique is also used to specialize method calls based on the class of the receiver, and when the identity of callees is known, type information can be propagated interprocedurally.

\subsection{Type Specialization}

As in prior works on LBBV [6, 7], YJIT implements a scheme to track and propagate type information, which is then used to specialize machine code. Table 1 outlines the types which are currently tracked by YJIT.

Table 1. Basic types tracked by YJIT.

\begin{tabular}{ll} 
Type & Description \\
\hline IMM & Immediate value of unknown type \\
FALSE & The immediate value false \\
NIL & The immediate value nil \\
TRUE & The immediate value true \\
FIXNUM & Immediate 63-bit signed integer \\
FLONUM & Immediate floating point value \\
STATIC_SYMBOL & Immediate Ruby symbol \\
\hline HEAP & Heap-allocated object of unknown type \\
ARRAY & Dynamically-extensible list \\
HASH & Map of keys to values \\
STRING & Character string
\end{tabular}

We note that this type specialization does not appear to have a measurable impact on performance at this point in time, either positive or negative. This is likely because execution time is dominated by bottlenecks in the CRuby implementation, such as inefficient method calls and instance variable accesses (see Section 6). Hence, we cannot yet make any claims about the impact of type specialization in this paper, but it is our belief that the impact will be positive once these inefficiencies are addressed.

\section{Evaluation}

A list of benchmarks used in our experiments is provided in Table 2, along with the number of instruction sequences (iseqs) compiled by YJIT to give an idea of scale and complexity. Methods which are never called are never compiled. We have intentionally avoided using microbenchmarks in our evaluation, and favored benchmarks that are based on realworld software. Our largest benchmarks are liquid-render, which renders Shopify's Liquid web template language, activerecord, which is based on the widely-used database access middleware, and railsbench, using the Ruby on Rails web framework.

The optcarrot benchmark is a synthetic benchmark that implements a Nintendo console emulator. We have included it because it is sizeable, and was considered an optimization target by the Ruby core developers in the development of CRuby for a long time. The Lee benchmark was written by Chris Seaton, key contributor on the TruffleRuby project, to experiment with transactional memory.

The 30k_methods and 30k_ifelse benchmarks are synthetic benchmarks written by us, designed to be a torture test for YJIT. These were not included for performance comparisons against other systems, but rather to assess whether large code size would lead to a collapse of performance due to instruction cache misses. Each benchmark features several thousand small methods that call each other in an acyclic call graph with depth 30 . The generated code is large enough that it cannot completely fit in the L1 instruction cache of any modern CPU. The 30k_ifelse benchmark has conditional branches to make branch prediction more challenging.

To increase the stability of our benchmark results, experiments were performed on AWS c5.metal dedicated instances with CPU frequency scaling disabled. The AWS instances used were running Ubuntu Linux 20.04 on Intel Xeon Cascade Lake processors. Each benchmark run was given 500 warmup iterations before 200 timing iterations were performed. Final results are averaged over 15 full runs for CRuby, YJIT, MJIT, and TruffleRuby.

Experiments with CRuby's MJIT used version 3.0.2, while experiments on YJIT were based on CRuby version 3.1.0dev, YJIT commit hash e9990eda $71^{2}$. We used a publicly released version of CRuby to benchmark MJIT because there are often performance bugs in prerelease versions. For benchmarks

\footnotetext{
${ }^{2}$ https://github.com/Shopify/yjit
} 
Table 2. Number of compiled instruction sequences (iseqs).

\begin{tabular}{|ccc|}
\hline Benchmark & Description & Compiled iseqs \\
\hline \hline 30k_ifelse & Calling over 9000 methods in a loop with conditionals & 9258 \\
\hline 30k_methods & Calling over 5000 methods in a loop & 5777 \\
\hline lee & Solving electronic circuit routing using Lee's algorithm & 71 \\
\hline optcarrot & Emulating a Nintendo NES console & 197 \\
\hline psych-load & Loading a large YAML file & 60 \\
\hline liquid-render & Rendering Liquid templates & 144 \\
\hline activerecord & Fetching from a SQLite database using ActiveRecord & 190 \\
\hline railsbench & Generating HTTP responses with a Rails application & 1381 \\
\hline
\end{tabular}

using MJIT, we specified the command-line arguments -jit-max-cache $=10000$ as well as $--j i t-m i n-c a l l s=10$ to enable it to reach the best peak performance possible. ${ }^{3}$ For experiments with TruffleRuby, version 21.2.0 of the JVM implementation was used as recommended by members of the TruffleRuby team, with the --jvm command-line switch.

\subsection{Performance on Benchmarks}

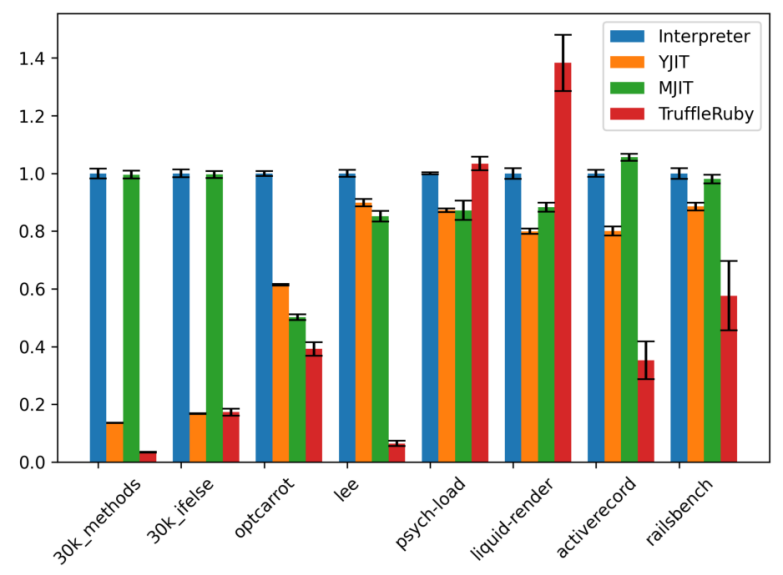

Figure 2. Mean execution time on benchmarks, normalized to the time taken by the interpreter (lower is better).

The results in Figure 2 show a comparison of the mean execution time after warmup for the CRuby interpreter, YJIT, MJIT, and TruffleRuby. Importantly, YJIT is always faster than the interpreter, which is not the case for MJIT. Even on railsbench, our most complex benchmark, YJIT achieves a speedup of $14 \%$ over the interpreter. Its performance compares favorably to that of MJIT, particularly when looking at benchmarks such as liquid-render, activerecord, and railsbench, which are all based on real-world software.

TruffleRuby, having the most sophisticated optimizations, and being a more mature JIT compiler, tends to have the best peak performance, though this is not the case on every

\footnotetext{
${ }^{3}$ https://k0kubun.medium.com/?p=756310f235a
}

benchmark. Notably, it tends to perform worse on benchmarks that call many native $\mathrm{C}$ functions, such as psych-load, as TruffleRuby uses an interpreter and JIT to run C extensions rather than directly running the native code. We also note that YJIT, which warms up very fast and predictably, produces performance numbers with very little variance. In contrast, TruffleRuby, presumably because it uses complex heuristics and background compilation threads, has nondeterministic warmup behavior, and sometimes produces numbers with high variance, as exemplified by railsbench.

The 30k_methods and 30k_ifelse benchmarks, which cannot entirely fit into the L1 instruction cache by design, clearly demonstrate that a large code size does not automatically translate into poor performance. LBBV generates linear code with more easy to predict branches, which helps the processor prefetching logic avoid costly i-cache misses.

\subsection{Warmup Time}

As shown in Table 3, the warmup time for YJIT is very fast, with clear speedups over the interpreter and MJIT even on the first iteration, and even on activerecord, which runs for less than 200ms. MJIT, which relies on an external C compiler in a background thread for compilation, has somewhat slower warmup. TruffleRuby, being that it initially relies on a tree-walking interpreter, has very high latency for the first many iterations, a problem which can get worse on larger programs. It also shows much more variance across time. It is a more sophisticated, but also less predictable VM.

Figure 3 is a plot of the time taken per iteration over the total run-time of the railsbench program, for the interpreter, MJIT, YJIT and TruffleRuby. This data is averaged over 6 independent runs for each VM. On this benchmark, MJIT yields a small speedup over the interpreter, on the order of 4\%. YJIT warms up almost instantly, and TruffleRuby takes slightly over 120 s to reach and then exceed the performance of YJIT. The latency for TruffleRuby is initially very high, which can be problematic for web applications. There is also a subsequent temporary loss of performance, again resulting in very high latency, presumably due to deoptimization. 
Table 3. Execution times at various iterations during the warmup process (lower is better) for 6 runs of every benchmark.

\begin{tabular}{|c|c|c|c|c|c|c|c|c|}
\hline Iter \# & $\begin{array}{c}\text { No-JIT } \\
\text { ActiveRecord }\end{array}$ & $\begin{array}{c}\text { YJIT } \\
\text { ActiveRecord }\end{array}$ & $\begin{array}{c}\text { MJIT } \\
\text { ActiveRecord }\end{array}$ & $\begin{array}{c}\text { Truffle } \\
\text { ActiveRecord }\end{array}$ & $\begin{array}{c}\text { No-JIT } \\
\text { Railsbench }\end{array}$ & $\begin{array}{c}\text { YJIT } \\
\text { Railsbench }\end{array}$ & $\begin{array}{c}\text { MJIT } \\
\text { Railsbench }\end{array}$ & $\begin{array}{c}\text { Truffle } \\
\text { Railsbench }\end{array}$ \\
\hline \hline 1 & $167 m s \pm 0.8 \%$ & $140 m s \pm 0.7 \%$ & $179 m s \pm 0.8 \%$ & $1.1 s \pm 2.7 \%$ & $3.8 s \pm 0.5 \%$ & $3.4 s \pm 0.9 \%$ & $3.7 s \pm 1.9 \%$ & $18.9 s \pm 3.4 \%$ \\
\hline 5 & $166 m s \pm 0.9 \%$ & $134 m s \pm 0.6 \%$ & $178 m s \pm 0.6 \%$ & $809 m s \pm 9.0 \%$ & $3.7 s \pm 1.2 \%$ & $3.3 s \pm 0.7 \%$ & $3.8 s \pm 3.0 \%$ & $5.4 s \pm 3.3 \%$ \\
\hline 10 & $166 m s \pm 0.8 \%$ & $133 m s \pm 0.6 \%$ & $177 m s \pm 1.4 \%$ & $486 m s \pm 14.6 \%$ & $3.8 s \pm 1.1 \%$ & $3.4 s \pm 1.8 \%$ & $3.8 s \pm 2.7 \%$ & $4.5 s \pm 7.1 \%$ \\
\hline 50 & $166 m s \pm 0.8 \%$ & $134 m s \pm 0.7 \%$ & $177 m s \pm 1.2 \%$ & $190 m s \pm 4.6 \%$ & $3.8 s \pm 0.9 \%$ & $3.4 s \pm 2.2 \%$ & $3.8 s \pm 2.5 \%$ & $2.9 s \pm 2.6 \%$ \\
\hline 100 & $166 m s \pm 0.7 \%$ & $134 m s \pm 0.7 \%$ & $178 m s \pm 1.8 \%$ & $154 m s \pm 14.1 \%$ & $3.9 s \pm 0.9 \%$ & $3.4 s \pm 1.6 \%$ & $3.8 s \pm 2.8 \%$ & $2.2 s \pm 2.3 \%$ \\
\hline 200 & $166 m s \pm 0.7 \%$ & $134 m s \pm 0.7 \%$ & $178 m s \pm 1.3 \%$ & $101 m s \pm 9.8 \%$ & $4.0 s \pm 1.1 \%$ & $3.5 s \pm 1.8 \%$ & $3.9 s \pm 1.4 \%$ & $2.4 s \pm 6.7 \%$ \\
\hline 500 & $166 m s \pm 0.8 \%$ & $134 m s \pm 0.6 \%$ & $177 m s \pm 1.4 \%$ & $60.4 m s \pm 14.6 \%$ & $4.0 s \pm 2.5 \%$ & $3.6 s \pm 1.2 \%$ & $3.9 s \pm 1.2 \%$ & $2.1 s \pm 8.6 \%$ \\
\hline
\end{tabular}

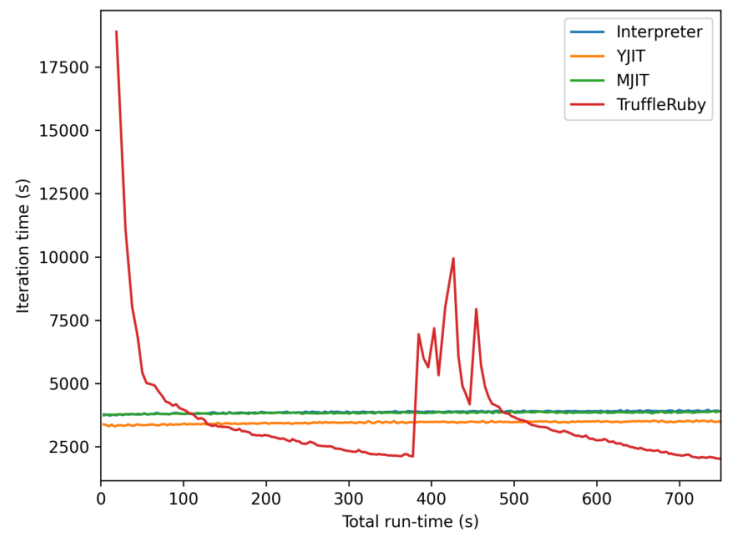

Figure 3. Time per iteration over total run-time averaged over 6 runs of one benchmark (lower is better).

\subsection{Code Size}

Table 4. Generated code size for each benchmark.

\begin{tabular}{|ccc|}
\hline Benchmark & Inline code size & Outlined code size \\
\hline \hline 30k_ifelse & $5.21 \mathrm{MiB}$ & $5.26 \mathrm{MiB}$ \\
\hline 30k_methods & $2.03 \mathrm{MiB}$ & $2.12 \mathrm{MiB}$ \\
\hline activerecord & $796 \mathrm{KiB}$ & $744 \mathrm{KiB}$ \\
\hline lee & $144 \mathrm{KiB}$ & $132 \mathrm{KiB}$ \\
\hline liquid-render & $337 \mathrm{KiB}$ & $317 \mathrm{KiB}$ \\
\hline optcarrot & $381 \mathrm{KiB}$ & $402 \mathrm{KiB}$ \\
\hline psych-load & $132 \mathrm{KiB}$ & $120 \mathrm{KiB}$ \\
\hline railsbench & $1.85 \mathrm{MiB}$ & $1.69 \mathrm{MiB}$ \\
\hline
\end{tabular}

In Table 4 we list the inline code size versus the outlined code size for each benchmark. The code size metrics give us a general sense of the scale and complexity of each benchmark. By keeping a separate outlined area for stubs and side-exits, we avoid interrupting the flow of code generated for method bodies. The 30k_ifelse benchmark has very large code size by design, and YJIT still delivers a very large performance gain, even though every single method gets executed in each iteration of the main benchmark loop, demonstrating that there is no direct correlation between generated code size and performance.

\section{Related Work}

Smalltalk \& Self Key ideas in dynamic language optimization, such as the use of inline caches and maps, which are now more commonly referred to as object shapes, can be traced back to Smalltalk [10] and Self [5].

Quickening is a technique for optimizing the performance of interpreters [4] by using inline caching to dispatch to specialized versions of bytecode instructions. This results in code which is easier for the compiler to optimize and has fewer unpredictable branches. YJIT leverages LBBV to achieve the same benefits and more, but quickening has the advantage that it is easier to implement and does not involve code generation.

Run-time Value Promotion The Psyco prototype for Python [13] proposed the notion of specializing an interpreter based on the running program. A key concept was the idea of promoting run-time values to compile-time so that code could be specialized based on these. This notion was later successfully adapted to JavaScript [8]. YJIT also implements run-time value promotion and uses it to specialize instance variable accesses and method calls.

PyPy and Meta-Tracing The PyPy project began as a followup to Psyco. PyPy $[2,3]$ uses a tracing JIT compiler to trace through a Python interpreter, itself written in an restricted subset of Python known as RPython [1]. This interpreter contains a few special annotations which help the tracing JIT unroll it into compiled and optimized native code. Lazy Basic Block Versioning is a novel kind of JIT compiler architecture first introduced in [6] and subsequently extended to propagate type information interprocedurally in [7]. The technique has been adapted for use in functional programming languages such as Scheme $[14,15]$ and to optimize gradual typing [12].

$\boldsymbol{J R} \boldsymbol{u} \boldsymbol{b} \boldsymbol{y}$ is a JVM-based implementation of Ruby $[16,23]$ which is still in active development. It leverages JVM features aimed at optimizing dynamic languages such as invokedynamic and offers very competitive performance results on some 
benchmarks. However, warmup times can be lengthy on larger benchmarks, and it greatly lacks when it comes to compatibility with existing Ruby code, because it does not support native Ruby gems, which are very common.

TruffleRuby is an implementation of Ruby based on the Truffle/Graal platform. The Truffle platform for building VMs bears some similarity to the idea of meta-tracing found in PyPy. An annotated interpreter is written for the source language, and this interpreter is automatically rewritten and compiled into optimized code using partial evaluation [21, 22]. TruffleRuby began by reusing code shared with JRuby, and eventually became an independent Ruby implementation. It benefits from many years of work on The Truffle platform, but also integrates optimizations that are specific to Ruby [9, 11, 18].

Stripe's Sorbet Compiler Stripe has recently announced that they will be open sourcing an ahead-of-time Ruby compiler $^{4}$ they have been working on. This compiler translates Ruby source files with additional Sorbet type annotations (gradual typing) into Ruby gems that integrate with the CRuby VM. Their motivations for building a compiler that integrates with CRuby include compatibility and ease of deployment, similarly to YJIT.

Other Ruby implementations There has been a surprisingly large number of projects aiming to implement Ruby JIT compilers and VMs, though most of these are no longer being maintaned. To name a few: Vidar Hokstad's Ruby compiler, IronRuby, LLRB, MacRuby, MagLev, Ruby OMR, RTL MJIT, Rubinius, RubyX, and Topaz.

\section{Future Work}

Faster Calling Convention Ruby is a programming language dominated by method calls. Basic constructs like loops to iterate over elements of an arrays are typically implemented as method calls with block arguments (closures) and most method bodies are very short. The way CRuby implements method calls is meant to be convenient for an interpreter, but it is clearly suboptimal for a JIT compiler. Each send instruction must set up a new control frame object that lives on a separate stack, and write multiple fields to it. We believe that the overhead from method calls currently dominates execution time. Inlining is possible in some cases, but in order to become performance-competitive, CRuby will need a faster calling convention, otherwise the current design will always impose an upper bound on the performance that can be achieved.

Object Shapes Similarly to method calls, the scheme used in CRuby for instance variable accesses is very costly, with multiple dynamic checks required for every read and write. This is likely to become a performance bottleneck. Modern dynamic language implementations have converged on the use of object shapes to solve this problem $[5,7,20]$ and we

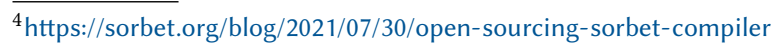

believe that the same solution can be used in CRuby to greatly improve the performance of instance variable accesses.

Optimizing Backend At the moment, YJIT directly generates machine code targetting x86-64. It would be desirable, both for the sake of portability, and to enable some simple low-level optimizations, to introduce some kind of intermediate representation between Ruby bytecode and the machine code we generate. Implementing this new level of abstraction could add some complexity to the system, and has the potential to slow down YJIT's warmup somewhat, but it may also lead to a codebase that is easier to maintain and better performance in the long run.

\section{Discussion \& Conclusion}

We have presented YJIT, an open source JIT compiler built inside CRuby. This compiler is based on an LBBV JIT compiler architecture, and, although it is still in early stages of development, our empirical data suggests that it succeeds in delivering fast warmup times and significant performance improvements over both the CRuby interpreter and MJIT.

YJIT is nearly $100 \%$ compatible with existing Ruby code and is able to match CRuby on all the tests in the Shopify and GitHub backend test suites. It has very fast and predictable warmup characteristics, delivering speedups on the first iteration, even for short-running benchmarks. Speedups from $15 \%$ to $19 \%$ over the CRuby interpreter were achieved on sizeable benchmarks based on real-world software.

Given that there are multiple obvious improvements that we can make to the design of YJIT, we have a clear path forward in terms of directions to pursue to enable YJIT to deliver much bigger speedups in the future.

\section{References}

[1] Davide Ancona, Massimo Ancona, Antonio Cuni, and Nicholas D. Matsakis. 2007. RPython: A Step towards Reconciling Dynamically and Statically Typed OO Languages. In Proceedings of the 2007 Symposium on Dynamic Languages (Montreal, Quebec, Canada) (DLS '07). Association for Computing Machinery, New York, NY, USA, 53-64. https://doi.org/10.1145/1297081.1297091

[2] Carl Friedrich Bolz, Antonio Cuni, Maciej Fijałkowski, Michael Leuschel, Samuele Pedroni, and Armin Rigo. 2011. Runtime Feedback in a Meta-Tracing JIT for Efficient Dynamic Languages. In Proceedings of the 6th Workshop on Implementation, Compilation, Optimization of Object-Oriented Languages, Programs and Systems (Lancaster, United Kingdom) (ICOOOLPS '11). Association for Computing Machinery, New York, NY, USA, Article 9, 8 pages. https: //doi.org/10.1145/2069172.2069181

[3] Carl Friedrich Bolz, Antonio Cuni, Maciej Fijalkowski, and Armin Rigo. 2009. Tracing the Meta-Level: PyPy's Tracing JIT Compiler. In Proceedings of the 4th Workshop on the Implementation, Compilation, Optimization of Object-Oriented Languages and Programming Systems (Genova, Italy) (ICOOOLPS '09). Association for Computing Machinery, New York, NY, USA, 18-25. https://doi.org/10.1145/1565824.1565827

[4] Stefan Brunthaler. 2010. Efficient Interpretation Using Quickening. SIGPLAN Not. 45, 12 (Oct. 2010), 1-14. https://doi.org/10.1145/1899661. 1869633 
[5] C. Chambers, D. Ungar, and E. Lee. 1989. An Efficient Implementation of SELF a Dynamically-Typed Object-Oriented Language Based on Prototypes. In Conference Proceedings on Object-Oriented Programming Systems, Languages and Applications (New Orleans, Louisiana, USA) (OOPSLA '89). Association for Computing Machinery, New York, NY, USA, 49-70. https://doi.org/10.1145/74877.74884

[6] Maxime Chevalier-Boisvert and Marc Feeley. 2015. Simple and Effective Type Check Removal through Lazy Basic Block Versioning. In 29th European Conference on Object-Oriented Programming, ECOOP 2015, July 5-10, 2015, Prague, Czech Republic (LIPIcs, Vol. 37), John Tang Boyland (Ed.). Schloss Dagstuhl - Leibniz-Zentrum für Informatik, 101-123. https://doi.org/10.4230/LIPIcs.ECOOP.2015.101

[7] Maxime Chevalier-Boisvert and Marc Feeley. 2016. Interprocedural Type Specialization of JavaScript Programs Without Type Analysis. In 30th European Conference on Object-Oriented Programming, ECOOP 2016, July 18-22, 2016, Rome, Italy (LIPIcs, Vol. 56), Shriram Krishnamurthi and Benjamin S. Lerner (Eds.). Schloss Dagstuhl - LeibnizZentrum für Informatik, 7:1-7:24. https://doi.org/10.4230/LIPIcs. ECOOP.2016.7

[8] Igor Costa, Péricles Alves, Henrique Nazaré Santos, and Fernando Magno Quintão Pereira. 2013. Just-in-time value specialization. In Proceedings of the 2013 IEEE/ACM International Symposium on Code Generation and Optimization (CGO). 1-11. https: //doi.org/10.1109/CGO.2013.6495006

[9] Benoit Daloze, Stefan Marr, Daniele Bonetta, and Hanspeter Mössenböck. 2016. Efficient and Thread-Safe Objects for DynamicallyTyped Languages. In Proceedings of the 2016 ACM SIGPLAN International Conference on Object-Oriented Programming, Systems, Languages, and Applications (Amsterdam, Netherlands) (OOPSLA 2016). Association for Computing Machinery, New York, NY, USA, 642-659. https://doi.org/10.1145/2983990.2984001

[10] L. Peter Deutsch and Allan M. Schiffman. 1984. Efficient Implementation of the Smalltalk-80 System. In Proceedings of the 11th ACM SIGACT-SIGPLAN Symposium on Principles of Programming Languages (Salt Lake City, Utah, USA) (POPL '84). Association for Computing Machinery, New York, NY, USA, 297-302. https://doi.org/10.1145/ 800017.800542

[11] Kevin Menard, Chris Seaton, and Benoit Daloze. 2018. Specializing Ropes for Ruby. In Proceedings of the 15th International Conference on Managed Languages \& Runtimes (Linz, Austria) (ManLang '18). Association for Computing Machinery, New York, NY, USA, Article 10, 7 pages. https://doi.org/10.1145/3237009.3237026

[12] Gregor Richards, Ellen Arteca, and Alexi Turcotte. 2017. The VM already knew that: leveraging compile-time knowledge to optimize gradual typing. Proc. ACM Program. Lang. 1, OOPSLA (2017), 55:155:27. https://doi.org/10.1145/3133879

[13] Armin Rigo. 2004. Representation-Based Just-in-Time Specialization and the Psyco Prototype for Python. In Proceedings of the 2004 ACM SIGPLAN Symposium on Partial Evaluation and Semantics-Based Program Manipulation (Verona, Italy) (PEPM '04). Association for Computing Machinery, New York, NY, USA, 15-26. https://doi.org/10.1145/ 1014007.1014010

[14] Baptiste Saleil and Marc Feeley. 2017. Interprocedural Specialization of Higher-Order Dynamic Languages Without Static Analysis. In 31st
European Conference on Object-Oriented Programming, ECOOP 2017, June 19-23, 2017, Barcelona, Spain (LIPIcs, Vol. 74), Peter Müller (Ed.). Schloss Dagstuhl - Leibniz-Zentrum für Informatik, 23:1-23:23. https: //doi.org/10.4230/LIPIcs.ECOOP.2017.23

[15] Baptiste Saleil and Marc Feeley. 2018. Building JIT compilers for dynamic languages with low development effort. In Proceedings of the 10th ACM SIGPLAN International Workshop on Virtual Machines and Intermediate Languages, VMIL@SPLASH 2018, Boston, MA, USA, November 4, 2018, Stephen Kell and Stefan Marr (Eds.). ACM, 36-46. https://doi.org/10.1145/3281287.3281294

[16] Aibek Sarimbekov, Andrej Podzimek, Lubomir Bulej, Yudi Zheng, Nathan Ricci, and Walter Binder. 2013. Characteristics of Dynamic JVM Languages. In Proceedings of the 7th ACM Workshop on Virtual Machines and Intermediate Languages (Indianapolis, Indiana, USA) (VMIL '13). Association for Computing Machinery, New York, NY, USA, 11-20. https://doi.org/10.1145/2542142.2542144

[17] Koichi Sasada. 2005. YARV: Yet Another RubyVM: Innovating the Ruby Interpreter. In Companion to the 20th Annual ACM SIGPLAN Conference on Object-Oriented Programming, Systems, Languages, and Applications (San Diego, CA, USA) (OOPSLA '05). Association for Computing Machinery, New York, NY, USA, 158-159. https://doi.org/10. 1145/1094855.1094912

[18] Chris Seaton. 2015. Specialising Dynamic Techniques for Implementing the Ruby Programming Language. Ph.D. Dissertation. University of Manchester.

[19] Pat. Shaughnessy. 2013 - 2014. Ruby under a microscope : An illustrated guide to ruby internals (1st edition ed.). No Starch Press, San Francisco.

[20] Andreas Wöß, Christian Wirth, Daniele Bonetta, Chris Seaton, Christian Humer, and Hanspeter Mössenböck. 2014. An Object Storage Model for the Truffle Language Implementation Framework. In Proceedings of the 2014 International Conference on Principles and Practices of Programming on the Fava Platform: Virtual Machines, Languages, and Tools (Cracow, Poland) (PPPF '14). Association for Computing Machinery, New York, NY, USA, 133-144. https://doi.org/10.1145/2647508. 2647517

[21] Thomas Würthinger, Christian Wimmer, Andreas Wöß, Lukas Stadler, Gilles Duboscq, Christian Humer, Gregor Richards, Doug Simon, and Mario Wolczko. 2013. One VM to Rule Them All. In Proceedings of the 2013 ACM International Symposium on New Ideas, New Paradigms, and Reflections on Programming \& Software (Indianapolis, Indiana, USA) (Onward! 2013). Association for Computing Machinery, New York, NY, USA, 187-204. https://doi.org/10.1145/2509578.2509581

[22] Thomas Würthinger, Andreas Wöß, Lukas Stadler, Gilles Duboscq, Doug Simon, and Christian Wimmer. 2012. Self-Optimizing AST Interpreters. In Proceedings of the 8th Symposium on Dynamic Languages (Tucson, Arizona, USA) (DLS '12). Association for Computing Machinery, New York, NY, USA, 73-82. https://doi.org/10.1145/2384577. 2384587

[23] Shijie Xu, David Bremner, and Daniel Heidinga. 2017. Fusing Method Handle Graphs for Efficient Dynamic JVM Language Implementations. In Proceedings of the 9th ACM SIGPLAN International Workshop on Virtual Machines and Intermediate Languages (Vancouver, BC, Canada) (VMIL 2017). Association for Computing Machinery, New York, NY, USA, 18-27. https://doi.org/10.1145/3141871.3141874 